is an analogue of histidine in which a methyl group is attached to one of the nitrogen atoms in the side chain. The authors observed that catalytic turnover for the modified enzyme (OE1) was higher than for $\mathrm{BH} 32$, an effect that they ascribed to more rapid hydrolysis of the acyl-enzyme intermediate.

Burke et al. then used directed evolution to optimize the function of Me-His in the enzyme's active site. A wide range of strategies was used to introduce mutations, ultimately resulting in the discovery of a variant, OE1.3, that had improved catalytic efficiency. This variant differed from OE1 by having six mutations, in which one amino-acid residue has been replaced by another. The authors found that OE1.3 hydrolyses a range of analogues of 2-phenylacetate esters in which only hydrogen atoms are attached to the carbon atom adjacent to the carbonyl $(\mathrm{C}=\mathrm{O})$ group in the molecules. However, analogues in which a methyl group is attached next to the carbonyl group were poor substrates. The authors therefore carried out further directed evolution to generate OE1.4, an enzyme that has improved catalytic activity with this class of substrate, and which predominantly hydrolyses one of the two mirror-image isomers of each substrate.

The Me-His residue in the modified enzymes acts as a nucleophilic catalyst that is broadly analogous to the nucleophilic residues found in serine hydrolase and cysteine hydrolase enzymes. But how might organocatalysis ${ }^{6}$ in general inspire the discovery of enzymes that are more distant from those found in nature? Organocatalysts speed up many different reactions using just a few generic mechanisms (activation modes), but the catalysis is often inefficient, requiring rather high catalyst loadings (typically 5-20 mole \%) ${ }^{6}$. Some of these activation modes are also widely used by enzymes; for example, enamine catalysis is used by class I aldolases ${ }^{7}$. But other activation modes are less widely used enzymatically, despite the fact that they can enable many potentially useful synthetic reactions.

Organocatalysts have been introduced into proteins in various ways, for example by using an attached biotin group as an anchor that binds to streptavidin ${ }^{8}$, or by chemically modifying genetically encoded unnatural amino-acid residues ${ }^{9}$. However, to realize the full power of an expanded range of catalytic chemical groups, substantial optimization is likely to be needed to generate catalytically efficient active sites. Burke et al. have shown that directed evolution can improve enzymes that contain an unnatural organocatalytic group. Their approach might also provide a route to efficient enzymes that use activation modes not found in nature, and which are much more efficient than organocatalysts themselves.

Adam Nelson is at the School of Chemistry and at the Astbury Centre for Structural Molecular Biology, University of Leeds,

Leeds LS2 9JT, UK.

e-mail:a.s.nelson@leeds.ac.uk

1. Burke, A. J. et al. Nature 570, 219-223 (2019).

2. Jeschek, M. et al. Nature 537, 661-665 (2016).

3. Heinisch, T. et al. Chem. Sci. 9, 5383-5388 (2018).

4. Yang, H. et al. Nature Chem. 10, 318-324 (2018).

5. Bjelic, S. et al. ACS Chem. Biol. 8, 749-757 (2013).

6. MacMillan, D. W. C. Nature 455, 304-308 (2008).

7. Windle, C., Müller, M., Nelson, A. \& Berry, A. Curr. Opin. Chem. Biol. 19, 25-33 (2014).

8. Nödling, A. R. et al. Angew. Chem. Int. Edn 57 12478-12482 (2018)

9. Drienovská, I., Mayer, C., Dulson, C. \& Roelfes, G. Nature Chem. 10, 946-952 (2018).

This article was published online on 27 May 2019.

\title{
Bacterial dormancy curbs phage epidemics
}

One type of CRISPR-Cas bacterial-defence system destroys phage and bacterial RNA, which leads to bacterial dormancy. Dormancy is found to limit viral spread, and also protects against unrelated viruses and viral mutants. See LETTER P.241

\section{SIMON A. JACKSON \& PETER C. FINERAN}

A $\mathrm{n}$ unseen war is being waged right under our noses, between microorganisms and their viral invaders. To fight the viruses called bacteriophages (phages) that target them, bacteria have evolved a diverse armoury of defences, which includes a range of protective immune systems called CRISPR-Cas.

On page 241, Meeske et al. ${ }^{1}$ reveal a curious twist in our understanding of the incredible variety of CRISPR-Cas defences, by demonstrating how a type of the CRISPR-Cas system that targets phage RNA protects bacteria from infection by DNA phages. The authors report that this CRISPR-Cas system responds to DNA phages by unleashing the destruction of both viral and bacterial RNA, which then causes infected bacterial cells to enter a state of dormancy that shuts down the cellular processes needed for viral replication. Meeske and colleagues reveal that this self-induced bacterial dormancy helps to suppress viral replication and viral outbreaks, including those caused by viral mutants that can escape other types of CRISPR-Cas defences, or viruses unrelated to the one that triggered dormancy. This defence response, in which the shutdown of an infected bacterial cell might benefit neighbouring bacteria, has interesting parallels with other types of defence system, such as bacterial abortive-infection

"These dormant
cells can act
as sacrificial
'decoy' cells."
systems or cell death in plants and animals that is induced by the innate branch of the immune system.

CRISPR-Cas systems are classified into six types termed I to VI. Most such systems capture and store short sequences of viral DNA as genetic 'memories' of phage invasion. These stored sequences are used to generate RNA guides that enable Cas enzymes to target and degrade viral DNA or RNA. Type VI systems are intriguing because they are the only ones that destroy viral RNA
(Fig. 1) rather than $\mathrm{DNA}^{2-5}$, yet most phages have DNA rather than RNA genomes.

Type VI CRISPR-Cas systems, which use a Cas enzyme called Cas13, have previously been shown ${ }^{2,3}$ to respond to infection by RNA viruses by activating a form of indiscriminate (low sequence specificity) RNA-degrading activity by Cas13. In addition, when bacteria were engineered so that Cas 13 targeted a messenger RNA encoded by a circular DNA sequence called a plasmid, bacterial growth was impaired ${ }^{2,6}$. This suggested that, in the absence of phage infection, Cas13 activation, and its indiscriminate RNA destruction, led to bacterial-cell dormancy. But what role dormancy has, if any, in the antiviral defence processes remained an unanswered question.

To address this issue, Meeske et al. studied a type VI defence system using the bacterium Listeria ivanovii and the DNA phage $\phi R R 4$. The authors engineered the type VI system to guide Cas13 to target different $\phi R R 4$ viral sequences and then analysed how effectively this system provided antiviral defence. Cas 13 did indeed provide defence when targeted to viral mRNAs and, surprisingly, protection was achieved regardless of whether or not the targeted viral mRNAs corresponded to genes that are essential for viral replication, or whether the genes were expressed early or late during viral infection. There was also extensive bacterial RNA degradation in the infected cells, which caused infected bacteria to enter a dormant state in which the bacterial cells were alive but could not replicate.

This form of Cas13-induced dormancy has considerable parallels with another class of phage-defence system called 
abortive-infection mechanisms ${ }^{7}$. If infected by viruses, bacteria harbouring abortive-infection systems enter a dormant state or trigger cell death, which provides a population-level antiviral defence.

Does Cas13-induced dormancy after viral infection offer the wider bacterial population a form of protection called herd immunity, in which resistant individuals help to slow the spread of infection to sensitive members of the population? Meeske and colleagues demonstrated that phage-infected bacteria possessing type VI immunity do indeed provide antiviral cross-protection to neighbouring cells of the same strain that lack any immunity against the infecting virus. The authors also observed that, by engineering Cas13-induced dormancy in uninfected cells, bacteria that suppressed viral epidemics were generated, probably because these dormant cells can act as sacrificial 'decoy' cells that viruses fail to infect successfully, which thereby depletes the viral population.

A study ${ }^{8}$ of type I-E CRISPR-Cas activity also reported bacterial-cell shutdown, but the effect was probably due to insufficient phage clearance, leading to a stalemate between bacterial defences and viral replication ${ }^{8}$. By contrast, Meeske et al. demonstrate that the bacterial dormancy induced by Cas 13 occurs through the active process of indiscriminate RNA degradation that is triggered following the recognition of viral RNA.

A challenge faced by CRISPR-Cas systems is if viral genetic mutants arise that escape recognition by the defence system. Meeske et al. showed that outbreaks of such mutant viruses are limited by the presence of wild-type viruses that cause Cas13-induced bacterial dormancy. Similarly, the authors found that when bacteria with type VI defences against $\phi R R 4$ were exposed to an unrelated virus, the level of infection by the unrelated virus was reduced if cells were also exposed to $\phi R R 4$. This type of broad-spectrum defence might offer advantages over CRISPR-Cas systems that do not induce dormancy and in which infection by viral mutants is unaffected by the presence of wild-type viruses.

The scarcity of type VI defence in nature compared with other CRISPR-Cas systems argues that the type VI strategy might not always be superior ${ }^{9}$. Indeed, other CRISPRCas systems have methods for dealing with mutant viruses, such as using feedback loops termed priming to update target memory ${ }^{10}$ or having enough flexibility to also recognize mutant forms of the viral target ${ }^{11}$. Probably, each type of strategy that fights back against viral mutants has different costs and benefits in particular ecological settings.

The Cas13-induced dormancy response has interesting implications for how type VI systems form memories of viral infections. Because viral DNA or RNA is needed to form CRISPR-Cas memories, viral infection and subsequent bacterial-cell survival

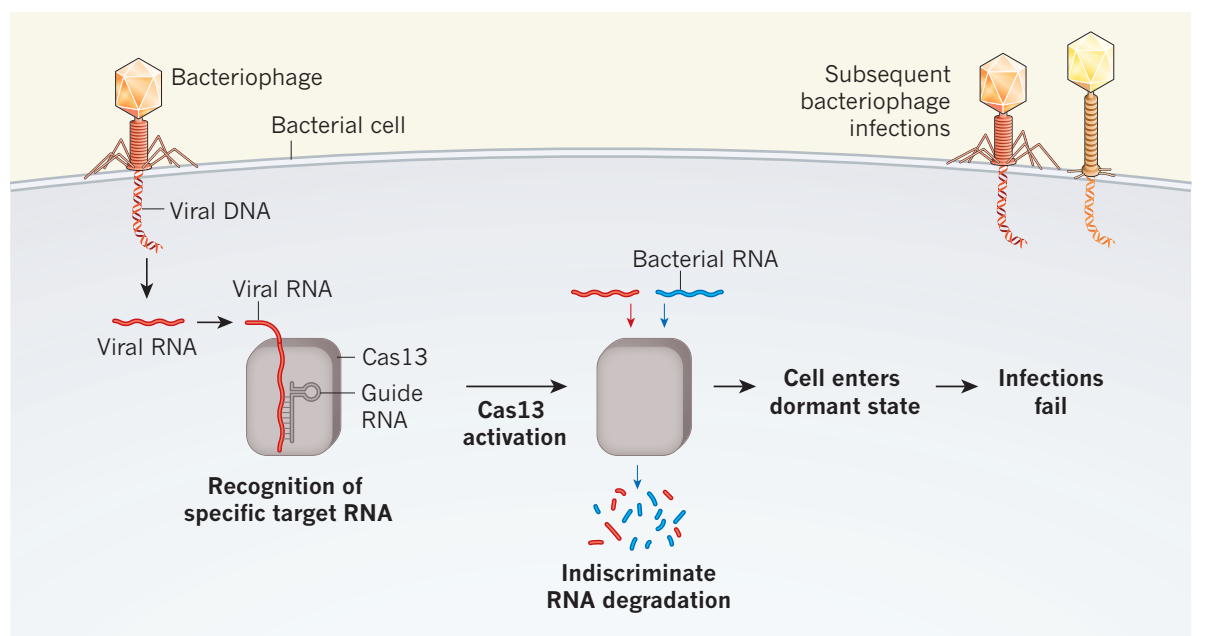

Figure 1 | A bacterial response to viral infection. Meeske et al. ${ }^{1}$ report how one type of antiviral defence system can protect bacterial populations against viruses called bacteriophages. In the antiviral bacterial defence system known as the type VI CRISPR-Cas system, a viral sequence can be recognized by bacteria if it matches a bacterially encoded guide RNA that fulfils the role of a bacterial 'memory' of a previous viral encounter. The protein Cas 13 binds a guide RNA and can destroy viral RNA that matches the guide sequence. The recognition of viral RNA then triggers the activation of indiscriminate RNA degradation by Cas 13 that destroys viral and bacterial RNA. This causes the cell to enter a dormant state that can result in the failure of viral infection. Importantly, if another virus, even if of a different type or a mutant virus, subsequently tries to infect the cell, infection fails because of cell dormancy. Such failed infection offers protection from viral infection for the entire bacterial population.

are necessary to update a type VI defence against viruses, including those not previously encountered, and mutated viruses ${ }^{10}$. In type II systems, failed infections by defective viruses can allow a memory update without causing cell death ${ }^{12}$. This might also offer a way for type VI systems to acquire new memories of viral infection. However, it would be more beneficial if some virus-infected cells clear the infection and recover from Cas13-induced dormancy. If dormancy helps bacteria to survive viral infection, it would be expected that the bacterial memories of DNA viruses stored by type VI systems would be biased towards targeting viral genes expressed early in the course of infection, thereby allowing the rapid suppression of viral infection and greater opportunity for bacterial recovery.

Intriguingly, the authors found that dormancy induced by targeting Cas 13 to a bacterial mRNA could be reversed in the absence of viral infection. However, whether or not a bacterial cell can survive viral infection probably depends on how far infection has progressed before dormancy is initiated. Bacterial dormancy triggered by certain RNA-targeting abortive-infection systems, such as ToxIN, is reversible in the absence of viral infection ${ }^{7}$, but it is unknown whether this is also the case for dormancy triggered by viral infection. Type III CRISPR-Cas systems activate enzymes that cause indiscriminate RNA destruction after the specific recognition of viral sequences, and this RNA destruction is inactivated when viral infection is suppressed ${ }^{13}$. If cells can 'wake' from the dormancy induced by the type VI system, then perhaps a similar system of temporal control of nonspecific RNA degradation might be responsible ${ }^{4,5}$. Alternatively, the activity of other bacterial factors or other antiviral defence systems might aid bacterial recovery after viral infection.

Meeske and colleagues' findings reinforce the idea that CRISPR-Cas defence systems are context-dependent. Further studies will be required to better understand how these diverse defence systems in different bacteria tackle the various bacteriophages that they naturally encounter.

Simon A. Jackson and Peter C. Fineran are in the Department of Microbiology \& Immunology, University of Otago, Dunedin 9054, New Zealand.

e-mail:peter.fineran@otago.ac.nz

1. Meeske, A. J., Nakandakari-Higa, S. \& Marraffini, L. A. Nature 570, 241-245 (2019).

2. Abudayyeh, O. O. et al. Science 353 , aaf5573 (2016).

3. East-Seletsky, A. et al. Nature 538, 270-273 (2016).

4. Smargon, A. A. et al. Mol. Cell 65, 618-630.e7 (2017).

5. Yan, W. X. et al. Mol. Cell 70, 327-339 (2018)

6. Meeske, A. J. \& Marraffini, L. A. Mol. Cell 71, 791-801 (2018).

7. Fineran, P. C. et al. Proc. Natl Acad. Sci. USA 106, 894-899 (2009).

8. Strotskaya, A. et al. Nucleic Acids Res. $\mathbf{4 5}$ 1946-1957 (2017).

9. Shmakov, S. et al. Mol. Cell 60, 385-397 (2015).

10.Jackson, S. A. et al. Science 356, eaal5056 (2017).

11.Pyenson, N. C. et al. Cell Host Microbe 22, 343-353 (2017).

12. Hynes, A. P., Villion, M. \& Moineau, S. Nature Commun. 5, 4399 (2014)

13.Athukoralage, J. S., Rouillon, C., Graham, S. Grüschow, S. \& White, M. F. Nature 562, 277-280 (2018).

This article was published online on 29 May 2019. 